\title{
Assessing prediction of diabetes in older adults using different adiposity measures: a 7 year prospective study in 6,923 older men and women
}

\author{
S. G. Wannamethee - O. Papacosta • P. H. Whincup • \\ C. Carson • M. C. Thomas • D. A. Lawlor • S. Ebrahim • \\ N. Sattar
}

Received: 24 August 2009 / Accepted: 23 December 2009/Published online: 10 February 2010

(C) The Author(s) 2010. This article is published with open access at Springerlink.com

\begin{abstract}
Aims/hypothesis The aim of this study was to examine whether waist circumference (WC) or WHR improve diabetes prediction beyond body mass index in older men and women, and to define optimal cut-off points.

Methods In this prospective study, non-diabetic men $(n=$ $3,519)$ and women $(n=3,404)$ aged $60-79$ years were followed up for 7 years. There were 169 and 128 incident cases of type 2 diabetes in men and women, respectively.
\end{abstract}

S. G. Wannamethee $(\bowtie) \cdot$ O. Papacosta $\cdot$ M. C. Thomas

Department of Primary Care and Population Health,

University College London Medical School,

Hampstead Campus, Rowland Hill St,

London NW3 2PF, UK

e-mail: goya@pcps.ucl.ac.uk

P. H. Whincup

Department of Community Health Sciences, St George's,

University of London,

London, UK

C. Carson $\cdot$ S. Ebrahim

Non-Communicable Disease Epidemiology Unit,

Department of Epidemiology and Population Health,

London School of Health and Tropical Medicine,

London, UK

D. A. Lawlor

MRC Centre for Causal Analyses in Translational Research,

Department of Social Medicine, University of Bristol,

Bristol, UK

N. Sattar

British Heart Foundation Glasgow Cardiovascular Research Centre,

Faculty of Medicine, University of Glasgow,

Glasgow, UK
Results BMI, WC and WHR all showed strong associations with incident type 2 diabetes independent of potential confounders. In men, the adjusted relative risks (top vs lowest quartile) were 4.71 (95\% CI 2.45-9.03) for BMI, 3.53 (95\% CI 1.92-6.48) for WC and 2.76 (95\% CI 1.58 4.82) for WHR. For women, the corresponding relative risks were 4.10 (95\% CI 2.16-7.79), 12.18 (95\% CI 4.83$30.74)$ and 5.61 (95\% CI 2.84-11.09) for BMI, WC and WHR, respectively. Receiver-operating characteristic curve analysis revealed similar associations for BMI and $\mathrm{WC}$ in predicting diabetes in men $(\mathrm{AUC}=0.726$ and 0.713 , respectively); WHR was the weakest predictor (AUC= 0.656). In women, $\mathrm{WC}$ was a significantly stronger predictor $(\mathrm{AUC}=0.780)$ than either $\mathrm{BMI}(\mathrm{AUC}=0.733)$ or WHR (AUC $=0.728 ; p<0.01$ for both). Inclusion of both $\mathrm{WC}$ and BMI did not improve prediction beyond BMI alone in men or WC alone in women. Optimal sensitivity and specificity for the prediction of type 2 diabetes was observed at a WC of $100 \mathrm{~cm}$ in men and $92 \mathrm{~cm}$ in women. Conclusions/interpretation In older men, BMI and WC yielded similar prediction of risk of type 2 diabetes, whereas WC was clearly a superior predictor in older women.

Keywords Abdominal adiposity - Body mass index · Type 2 diabetes - Waist circumference $\cdot$ Waist-to-hip ratio
Abbreviations
ARIC Atherosclerosis Risk in Communities
BHF British Heart Foundation
CHD Coronary heart disease
ROC Receiver-operating characteristic
WC Waist circumference 


\section{Introduction}

Obesity is an important risk factor for type 2 diabetes. The strong association between abdominal fat and increased risk of cardio-metabolic risk [1] suggests that measures of central fat distribution such as waist circumference (WC) and WHR may be better than measures of general obesity, such as BMI, in predicting type 2 diabetes. This may be particularly important in the elderly population in whom BMI is known to be a weaker indicator of obesity because visceral fat increases and muscle mass declines with age [2]. The National Heart Lung and Blood Institute recommends the use of WC as an additional means of assessing the risks associated with excessive adiposity [3]. Although several prospective studies have shown WC to be a better predictor of diabetes than BMI [4-8], the results are inconclusive [9-13]. Indeed, in a recent meta-analysis based on 32 studies, BMI, WC and WHR were all shown to have similar associations with risk of incident type 2 diabetes, suggesting that all measures are important in predicting the risk of diabetes [14]. However, this metaanalysis did not stratify analyses by sex, which may be a potentially important omission. Furthermore, few studies have compared the use of these anthropometric markers in older individuals and determined whether the use of BMI combined with measures of body fat distribution (WC or WHR) improves the ability to predict diabetes. This is an important clinical question as the greatest relative increase in type 2 diabetes prevalence in developed countries in the next 25 years will be in those aged over 65 years [15]. Uncertainties also remain about the best combination of anthropometric measures to use in men and women and the optimal cut-off points, particularly in older people.

We have examined the predictive power of BMI, WC and WHR in predicting incident diabetes and evaluated WC cut-off points to assess diabetes risk in a prospective study of men and women aged 60-79 years. We have also examined the combined use of BMI and measures of fat distribution (WC and WHR) in predicting type 2 diabetes.

\section{Methods}

Data from the British Regional Heart Study and the British Women's Heart and Health Study were used. The British Regional Heart Study is a prospective study of cardiovascular disease involving 7,735 British men drawn from general practices in 24 British towns and cities who were followed up from 1978 to 1980 [16, 17]. In 1998-2000, all surviving men, now aged 60-79 years, were invited for a 20th year follow-up examination; 4,252 men $(77 \%$ of survivors) attended for examination. In 1999-2001, a parallel study of 4,286 women $(60 \%$ of those invited) of the same age and drawn from 22 of the same 24 towns and cities was established, with the addition of one more study city (Bristol) [18]. Full details of the selection of participants and measurements have been reported previously [18]. The study population was predominantly $(>95 \%)$ described as white by examining nurses.

Similar protocols for data collection were used in both studies. In both studies, nurses administered questionnaires, made physical measurements and collected fasting venous blood samples, from which serum was stored at $-70^{\circ} \mathrm{C}$ for subsequent analysis. Men and women completed detailed questionnaires on: medical history; medication; and lifestyle, including cigarette smoking, alcohol consumption and physical activity. Details of measurement and classification methods for smoking status, physical activity, social class, blood pressure and blood lipids in the two cohorts have been described elsewhere [18-21]. All participants provided written informed consent to the investigation and ethical approval was provided by all relevant local research ethics committees. Fasting glucose measurements were available in 3829 women (89\%) and 4032 men (95\%). We excluded all men and women with prevalent diabetes (defined as the presence of a diagnosis by a doctor of diabetes and/or a fasting glucose of $\geq 7 \mathrm{mmol} / \mathrm{l}) \quad(n=481$ men, $n=377$ women) and four women whose diabetes status could not be determined. We further excluded those with missing BMI, waist and hip measurements $(n=32$ men; $n=44$ women). After these exclusions, a total of 3519 non-diabetic men and 3404 non-diabetic women were eligible for the current study.

Anthropometric measurements Measurements included height, weight and waist and hip circumferences. Participants were measured standing in light clothing without shoes. Height was measured with a Holtain (Crosswell, UK) stadiometer to the last complete $0.1 \mathrm{~cm}$ and weight was measured with a Soehnle (Waagen, Murrhardt, Germany) digital electronic scale to the last complete $0.1 \mathrm{~kg}$. Body mass index (weight $/$ height $^{2}$ in $\mathrm{kg} / \mathrm{m}^{2}$ ) was calculated for each individual. Waist and hip circumferences were measured in duplicate with an insertion tape (CMS, London, UK); hip circumference was measured at the point of maximum circumference over the buttocks. The waist measurement was taken from the midpoint between the iliac crest and the lower ribs measured at the sides. WHR was calculated as WC divided by hip circumference.

Follow-up All men and women were followed up for allcause mortality, cardiovascular morbidity and development of type 2 diabetes. Losses to follow-up remained exceptionally low $(<3 \%)$ in both cohorts. The analysis is based on follow-up from re-screening (1998-2000) to June 2006 in men and from 1999-2001 to September 2007 in women, 
a mean follow-up period of 7 years (6-8 years). Information on deaths has been collected through the established 'tagging' procedures provided by the National Health Service central registers. Information on the incidence of new events has been obtained from the combination of regular two yearly reviews of patients' notes (including hospital and clinic correspondence) through to the end of the study period and from personal questionnaires to surviving participants, repeated since the initial examination, asking whether a doctor had ever told them that they had diabetes, the year of diagnosis and reported use of medications specifically for diabetes. Self-report of doctordiagnosed diabetes has shown to be a valid measure of recording diabetes in the present study [22]. The medicalrecord review was compared with the patient's recall of doctordiagnosed diabetes. In $97 \%$ of men who reported a doctor diagnosis of diabetes on the questionnaire, the diagnosis was confirmed on review of the records. Because of the high agreement, self-report of diabetes has been included.

Statistical analysis The study population was stratified into sex-specific quartiles of BMI, WC and WHR. A Cox proportional hazards model was used to assess age-adjusted and multivariate-adjusted hazard ratio (HR). Age, systolic blood pressure, log triacylglycerol and log fasting glucose were fitted as continuous variables in the multivariate analysis. Physical activity, smoking, parental history of diabetes, manual social class, pre-existing CHD and alcohol intake were fitted as categorical variables. Finally, receiving-operating characteristic (ROC) curve analyses and the respective AUC [23] were used to compare the predictive power of baseline WC, WHR, and BMI on risk of type 2 diabetes in men and women. All data analyses were performed with STATA version 10.0.

\section{Results}

During follow-up, there were 165 incident cases of type 2 diabetes in the 3,519 men $(7.15 / 1,000$ person-years $)$ and 128 cases in the 3,404 women (5.40/1,000 person-years). BMI correlated most strongly with WC in both men and women ( $r=0.87$ and $r=0.83$, respectively) and correlated more with WHR in men than in women $(r=0.53$ and $r=$ 0.35 ). Sex-specific baseline characteristics of the study sample are shown in Table 1. Men have higher WC and WHR than women. Mean age, BMI and systolic blood pressure were similar for men and women. Compared with the women, men were more physically active and had higher rates of smoking, consumption of alcohol and preexisting CHD, but lower rates of family history of diabetes.

The relationships between WC, WHR and BMI and risk of type 2 diabetes in men and women are shown in Table 2.
All indicators were strongly and significantly associated with risk of type 2 diabetes in both men and women after adjustment for potential confounders including age, social class, alcohol intake, smoking status, physical activity, parental history of diabetes and pre-existing CHD. Further adjustment for systolic blood pressure, HDL-cholesterol and log plasma glucose reduced the associations, but they remained significant. In men BMI and WC showed similar associations; WHR was the weakest predictor. In women WC was by far the strongest predictor.

The positive association between BMI and the risk of type 2 diabetes remained strong even after adjustment for WHR in both men and women ( $p$ for trend $<0.0001$ for both sexes; Tables 2, 3). In men BMI was a significant predictor of diabetes after adjustment for age and $\mathrm{WC}$ and remained significant even after additional adjustment for other potential confounders and possible mediators $(p=0.01)$. However, in women BMI was not a predictor of diabetes when adjustments were made for age and $\mathrm{WC}$ alone. By contrast WC remained significantly associated with diabetes in women in the fully adjusted model that included adjustment for BMI. In men adjustment for BMI attenuated the association between $\mathrm{WC}$ and diabetes in men and this association was not significant after adjustment for potential confounders and mediators. Adjustment for BMI attenuated the association between WHR and diabetes in men but made little difference in women.

There was no significant interaction between age or parental history and adiposity measures with risk of diabetes and no significant interaction was seen between WC and BMI and diabetes in men or women ( $p=0.49$ and $p=0.77$ for men and women, respectively).

Unadjusted ROC analyses indicate that BMI and WC were similar in predicting type 2 diabetes in men; WHR was the weakest (Fig. 1). In men, the AUC was 0.726 for BMI, 0.713 for WC ( $p=0.20$ for differences in AUC for $\mathrm{WC}$ vs $\mathrm{BMI}$ ) and $\mathrm{AUC}=0.656$ for WHR. Addition of WC to $\mathrm{BMI}$ did not improve prediction in men (difference in AUC $=0.001 p=0.73$ ). When stratified by BMI levels $\left(<25 \mathrm{~kg} / \mathrm{m}^{2}, 25-29.9 \mathrm{~kg} / \mathrm{m}^{2}\right.$ and $\left.30+\mathrm{kg} / \mathrm{m}^{2}\right), \mathrm{WC}$ did not improve prediction of diabetes in any of the BMI groups. In women, WC was the best predictor of type 2 diabetes $(\mathrm{AUC}=0.780)$ and was significantly better than BMI (AUC $=$ $0.733, p=0.002)$ and WHR (AUC $=0.728, p=0.005)$. WC improved prediction within all BMI groups, particularly those who were not obese (BMI $<30 \mathrm{~kg} / \mathrm{m}^{2}$ ). The addition of BMI or WHR to WC did not improve prediction in women (difference in $\mathrm{AUC}=0.0007, p=0.73$ for adding $\mathrm{BMI}$; difference in $\mathrm{AUC}=0.008, p=0.63$ for adding WHR). Adjustment for age made little difference to the ROC curves.

We also assessed the improvement of prediction of the anthropometric measures when age and parental history were included in the model. In both men and women BMI 
Table 1 Baseline characteristics in men and women with no prevalent diabetes

Data are mean values (SD) unless otherwise stated

${ }^{a}$ Incident cases of diabetes: 165

${ }^{\mathrm{b}}$ Incident cases of diabetes: 128

${ }^{\mathrm{c}}$ Geometric mean (interquartile range)

\begin{tabular}{|c|c|c|c|}
\hline Characteristic & $\operatorname{Men}^{\mathrm{a}}(n=3,519)$ & Women $^{\mathrm{b}}(n=3,404)$ & $p$ value \\
\hline Age (years) & $68.6(5.5)$ & $69.3(5.5)$ & $<0.0001$ \\
\hline BMI $\left(\mathrm{kg} / \mathrm{m}^{2}\right)$ & $26.7(3.5)$ & $27.3(4.8)$ & $<0.0001$ \\
\hline WC (cm) & $96.5(10.0)$ & $85.4(11.7)$ & $<0.0001$ \\
\hline Hip circumference $(\mathrm{cm})$ & $102.1(6.9)$ & $104.6(10.0)$ & $<0.0001$ \\
\hline WHR & $0.94(0.06)$ & $0.81(0.07)$ & $<0.0001$ \\
\hline Inactive, $\%(n)$ & $32.6(1109)$ & $63.0(2065)$ & $<0.0001$ \\
\hline Current smokers, \% $(n)$ & $13.0(455)$ & $11.05(376)$ & 0.0127 \\
\hline Parental history of diabetes, $\%(n)$ & $5.5(195)$ & $9.3(318)$ & $<0.0001$ \\
\hline Manual social class, $\%(n)$ & $50.7(1784)$ & $48.9(1518)$ & 0.1439 \\
\hline Systolic blood pressure (mmHg) & $148.5(23.9)$ & $146.2(24.9)$ & 0.0001 \\
\hline HDL-cholesterol (mmol/l) & $1.34(0.34)$ & $1.67(0.45)$ & $<0.0001$ \\
\hline Triacylglycerol $^{\mathrm{c}}(\mathrm{mmol} / \mathrm{l})$ & $1.58(1.13,2.15)$ & $1.62(1.2,2.16)$ & 0.0549 \\
\hline Glucose $^{c}(\mathrm{mmol} / \mathrm{l})$ & $5.55(5.52,5.91)$ & $5.68(5.4,6)$ & $<0.0001$ \\
\hline Pre-existing CHD, \% $(n)$ & $10.7(375)$ & $15.3(515)$ & $<0.0001$ \\
\hline Daily and weekend drinking, \% (n) & $63.6(2192)$ & 38.89 (1216) & $<0.0001$ \\
\hline
\end{tabular}

Table 2 Age-adjusted and multivariate-adjusted hazard ratios and 95\% CI for type 2 diabetes according to quartiles of anthropometric indicators in men

\begin{tabular}{|c|c|c|c|c|c|c|c|}
\hline Variable & Cases & $\begin{array}{l}\text { Rate/1,000 } \\
\text { person-years }\end{array}$ & Age-adjusted & Adjusted $^{\mathrm{a}}$ & Adjusted $^{\mathrm{b}}$ & Adjusted $^{\mathrm{c}}$ & Adjusted $^{\mathrm{d}}$ \\
\hline All men & 165 & 7.15 & & & & & \\
\hline \multicolumn{8}{|l|}{ Quartiles } \\
\hline \multicolumn{8}{|l|}{ BMI $\left(\mathrm{kg} / \mathrm{m}^{2}\right)$} \\
\hline$<24.38$ & 11 & 1.94 & 1.00 & 1.00 & 1.00 & 1.00 & 1.00 \\
\hline $24.38-$ & 22 & 3.76 & $1.94(0.94-4.00)$ & $1.85(0.89-3.81)$ & $1.72(0.83-3.57)$ & $1.40(0.67-2.92)$ & $1.43(0.68-2.99)$ \\
\hline $26.42-$ & 38 & 6.40 & $3.29(1.68-6.43)$ & $3.04(1.55-5.96)$ & $2.21(1.11-4.39)$ & $1.88(0.92-3.83)$ & $1.57(0.77-3.23)$ \\
\hline $28.71-$ & 94 & 16.82 & $8.90(4.76-16.6)$ & $7.35(3.90-13.84)$ & $4.71(2.45-9.03)$ & $3.36(1.56-7.21)$ & $2.48(1.14-5.42)$ \\
\hline$p$ trend quartiles & & & $<0.001$ & $<0.001$ & $<0.001$ & $<0.001$ & 0.014 \\
\hline \multicolumn{8}{|l|}{ WC (cm) } \\
\hline$<90.1$ & 13 & 2.23 & 1.00 & 1.00 & 1.00 & 1.00 & 1.00 \\
\hline $90.1-$ & 27 & 4.60 & $2.08(1.07-4.02)$ & $1.95(1.00-3.78)$ & $1.52(0.78-2.97)$ & $1.48(0.76-2.89)$ & $1.27(0.65-2.48)$ \\
\hline $96.5-$ & 40 & 6.86 & $3.1(1.66-5.8)$ & $2.84(1.52-5.33)$ & $1.93(1.02-3.65)$ & $1.69(0.89-3.24)$ & $1.31(0.68-2.54)$ \\
\hline $102.5-$ & 85 & 15.35 & $7.06(3.94-12.65)$ & $5.89(3.26-10.65)$ & $3.53(1.92-6.48)$ & $2.17(1.08-4.37)$ & $1.59(0.77-3.26)$ \\
\hline$p$ trend quartiles & & & $<0.001$ & $<0.001$ & $<0.001$ & 0.026 & 0.214 \\
\hline \multicolumn{8}{|l|}{ WHR } \\
\hline$<0.907$ & 16 & 2.7 & 1.00 & 1.00 & 1.00 & 1.00 & 1.00 \\
\hline $0.907-$ & 35 & 6.00 & $2.25(1.25-4.07)$ & $2.03(1.12-3.69)$ & $1.64(0.9-2.97)$ & $1.53(0.84-2.79)$ & $1.26(0.68-2.3)$ \\
\hline $0.95-$ & 42 & 7.23 & $2.74(1.54-4.88)$ & $2.41(1.35-4.32)$ & $1.75(0.98-3.15)$ & $1.46(0.81-1.27)$ & $1.20(0.66-2.19)$ \\
\hline $0.98-$ & 72 & 13.13 & $5.10(2.97-8.78)$ & $4.38(2.53-7.59)$ & $2.76(1.58-4.82)$ & $2.25(1.27-3.98)$ & $1.65(0.92-2.95)$ \\
\hline$p$ trend quartiles & & & $<0.001$ & $<0.001$ & $<0.001$ & 0.03 & 0.069 \\
\hline
\end{tabular}

${ }^{a}$ Adjusted for age, smoking, physical activity, alcohol intake, parental history of diabetes and pre-existing CHD

${ }^{\mathrm{b}}$ Adjusted for age, smoking, physical activity, alcohol intake, parental history of diabetes, pre-existing CHD, systolic blood pressure, HDL-C and $\log$ blood glucose

${ }^{\mathrm{c}}$ Adjusted for age and BMI/WC

${ }^{\mathrm{d}}$ Adjusted for age, smoking, physical activity, alcohol intake, parental history of diabetes, pre-existing CHD, systolic blood pressure, HDL-C, log blood glucose and BMI/WC 
Table 3 Age-adjusted and multivariate-adjusted hazard ratio and 95\% CI for type 2 diabetes according to quartiles of anthropometric indicators in women

\begin{tabular}{|c|c|c|c|c|c|c|c|}
\hline Variable & Cases & $\begin{array}{l}\text { Rate/1,000 } \\
\text { person-years }\end{array}$ & Age-adjusted & Adjusted $^{\mathrm{a}}$ & Adjusted $^{\mathrm{b}}$ & Adjusted $^{\mathrm{c}}$ & Adjusted $^{\mathrm{d}}$ \\
\hline All women & 128 & 5.40 & & & & & \\
\hline \multicolumn{8}{|l|}{ Quartiles } \\
\hline \multicolumn{8}{|l|}{ BMI $\left(\mathrm{kg} / \mathrm{m}^{2}\right)$} \\
\hline$<24.07$ & 12 & 2.03 & 1.00 & 1.00 & 1.00 & 1.00 & 1.00 \\
\hline $24.07-$ & 12 & 2.01 & $0.98(0.44-2.17)$ & $0.99(0.44-2.20)$ & $0.86(0.38-1.92)$ & $0.64(0.29-1.44)$ & $0.64(0.28-1.44)$ \\
\hline $26.65-$ & 26 & 4.35 & $2.14(1.08-4.23)$ & $2.01(1.01-4.00)$ & $1.64(0.82-3.28)$ & $0.94(0.46-1.94)$ & $0.92(0.44-1.91)$ \\
\hline $29.78-$ & 78 & 13.3 & $6.46(3.52-11.88)$ & $5.80(3.11-10.81)$ & $4.10(2.16-7.79)$ & $1.34(0.61-2.92)$ & $1.27(0.57-2.82)$ \\
\hline$p$ trend quartiles & & & $<0.001$ & $<0.001$ & $<0.001$ & 0.162 & 0.231 \\
\hline \multicolumn{8}{|l|}{$\mathrm{WC}(\mathrm{cm})$} \\
\hline$<77.1$ & 5 & 0.84 & 1.00 & 1.00 & 1.00 & 1.00 & 1.00 \\
\hline $77.1-$ & 13 & 2.16 & $2.60(0.93-7.28)$ & $2.54(0.90-7.12)$ & $2.30(0.81-6.46)$ & $2.40(0.85-6.76)$ & $2.22(0.79-6.29)$ \\
\hline $84.05-$ & 23 & 3.86 & $4.68(1.78-12.3)$ & $4.34(1.64-11.44)$ & $3.83(1.44-10.16)$ & $4.00(1.49-10.77)$ & $3.60(1.33-9.73)$ \\
\hline $92.3-$ & 87 & 15.12 & $18.34(7.44-45.16)$ & $16.64(6.70-41.35)$ & $12.18(4.83-30.74)$ & $13.68(5.11-36.66)$ & $10.82(3.98-29.37)$ \\
\hline$p$ trend quartiles & & & $<0.001$ & $<0.001$ & $<0.001$ & $<0.001$ & $<0.001$ \\
\hline \multicolumn{8}{|l|}{ WHR } \\
\hline$<0.77$ & 10 & 1.64 & 1.00 & 1.00 & 1.00 & 1.00 & 1.00 \\
\hline $0.77-$ & 12 & 1.99 & $1.25(0.54-2.9)$ & $1.24(0.54-2.88)$ & $1.08(0.46-2.54)$ & $1.05(0.45-2.43)$ & $0.95(0.4-2.24)$ \\
\hline $0.81-$ & 33 & 5.61 & $3.61(1.78-7.32)$ & $3.29(1.62-6.69)$ & $2.85(1.4-5.82)$ & $2.56(1.25-5.23)$ & $2.3(1.12-4.74)$ \\
\hline $0.86-$ & 73 & 12.83 & $8.39(4.33-16.27)$ & $7.36(3.78-14.36)$ & $5.61(2.84-11.09)$ & $5.38(2.75-10.52)$ & $4.25(2.13-8.47)$ \\
\hline$p$ trend quartiles & & & $<0.001$ & $<0.001$ & $<0.001$ & $<0.001$ & $<0.001$ \\
\hline
\end{tabular}

${ }^{a}$ Adjusted for age, smoking, physical activity, alcohol intake, parental history of diabetes and pre-existing CHD

${ }^{\mathrm{b}}$ Adjusted for age, smoking, physical activity, alcohol intake, parental history of diabetes, pre-existing CHD, systolic blood pressure, HDL-C and log blood glucose

${ }^{\mathrm{c}}$ Adjusted for age and BMI/WC

${ }^{\mathrm{d}}$ Adjusted for age, smoking, physical activity, alcohol intake, parental history of diabetes, pre-existing CHD, systolic blood pressure, HDL-C, log blood glucose and BMI/WC

and WC significantly improved prediction of diabetes beyond age and parental history alone. The AUC for a model with age and parental history was 0.52 for men. This increased significantly to 0.73 with the addition of BMI and 0.71 with WC (for all differences in AUC $p<0.0001$ ). The addition of WC made no significant improvement beyond BMI. For women the AUC for age and parental history was 0.60 and this improved significantly with the addition of $\mathrm{BMI}(\mathrm{AUC}=0.75)$ or $\mathrm{WC}(\mathrm{AUC}=0.79$; for all differences in AUC $p<0.0001)$. The addition of WC to the model with age, parental history and BMI improved the prediction in women $(\mathrm{AUC}=0.79, p=0.01)$.

We also assessed the optimal cut-off points for BMI and WC for identifying diabetes in men and women. Table 4 shows the sensitivity, specificity and positive predictive value for the various cut-off points for BMI and WC in men and women. For women, $92 \mathrm{~cm}$ appeared to be the optimal WC cut-off point in terms of the sum of the sensitivity and specificity; for men, the optimal cut-off point for WC was $100 \mathrm{~cm}$. For BMI the optimal cut-off point for men was between 28 and $29 \mathrm{~kg} / \mathrm{m}^{2}$ and for women the optimal cutoff point was between 29 and $30 \mathrm{~kg} / \mathrm{m}^{2}$.

\section{Discussion}

In this study of older men and women, aged 60-79 years, both general adiposity (BMI) and the common measures of regional adiposity (WC and WHR) strongly and independently predicted incident diabetes in both men and women after adjusting for potential confounders and risk factors, including plasma lipids, plasma glucose and blood pressure. Our study confirms previous studies showing that both overall and abdominal adiposity play an important role in diabetes [4-14] and extends these findings to older adults. There has been much discussion of which anthropometric measure best predicts diabetes and the usefulness of WC or WHR over BMI [14]. Few prospective studies have examined these issues in older adults. Our study also provides new information regarding the possible optimal 

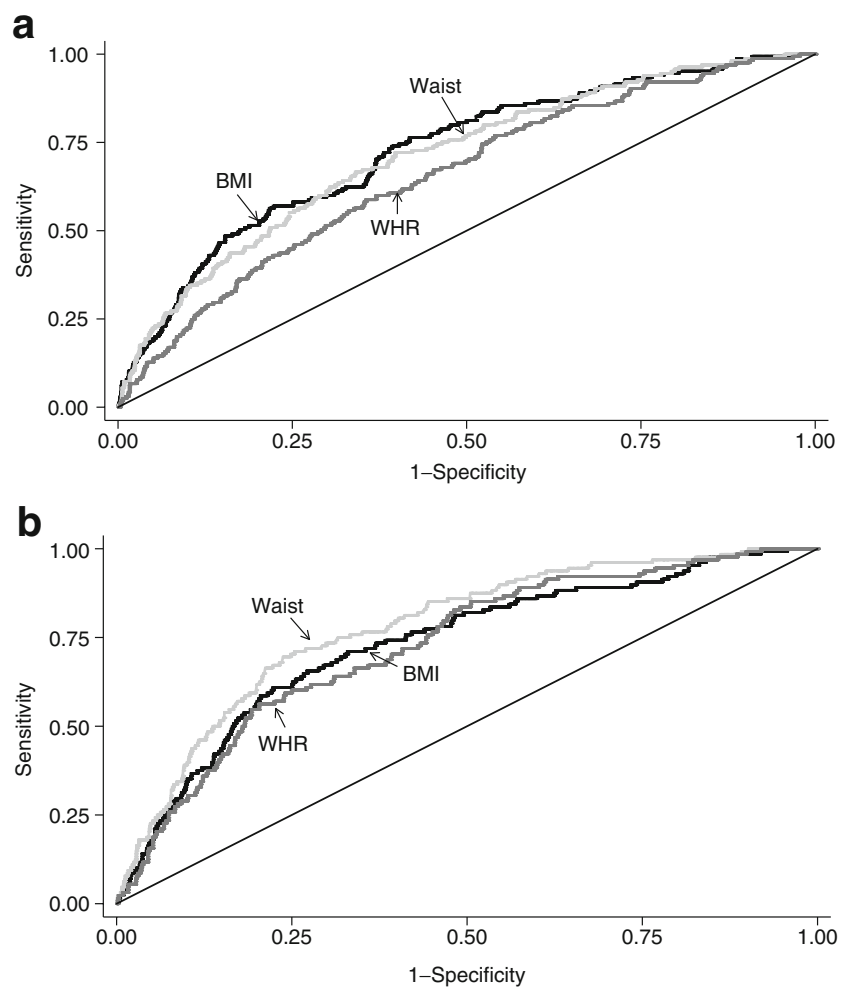

Fig. 1 The ROC curves (unadjusted) for BMI, WC and WHR for (a) men and (b) women. ROC area in men: BMI 0.726; WHR 0.656; WC 0.713. ROC area in women: BMI 0.733; WHR 0.728; WC 0.780

range of clinical utility for $\mathrm{WC}$ in predicting future diabetes in older adults. By analysis of ROC curves we have shown that in men, WC and BMI showed similar associations with incident diabetes; WHR was the weakest predictor. However, in women the WC was a significantly better indicator of diabetes risk than BMI or WHR, and enhanced prediction of diabetes beyond BMI.

There is no clear consensus on which obesity measure performs best in predicting diabetes. Previous prospective studies comparing WC, BMI and WHR for predicting diabetes risk have been inconsistent [4-13]. Indeed, in a recent meta-analysis of 32 studies worldwide it was concluded that these three obesity indicators have similar associations with incident diabetes [14]. Most of the studies included were carried out in populations with wide ageranges and analyses but, critically, the analyses were not stratified by sex or age group. Our finding that $\mathrm{WC}$ is a better predictor of diabetes than BMI or WHR in older women but not in older men is consistent with and extends data from several other prospective studies. The Iowa Women's Health Study of postmenopausal women (aged 55-69 years) showed those in the top quintile of the WC distribution to be associated with the largest relative risk compared with the top quintile of BMI and WHR [7]. In the MONICA/KORA Augsburg Cohort Study, WC emerged as the stronger predictor of type 2 diabetes in women but in men BMI appeared to be the stronger predictor, as was seen in our study [11]. In the Nurses Health Study of women aged 30-55 years, WC was the strongest predictor of diabetes when compared with BMI and WHR [8]. In the Health Professionals Follow-Up Study of men aged 4075 years, BMI and WC showed similar associations; WHR was the weakest predictor [10]. In the EPIC-Potsdam Study of men and women aged 35-65 years WC was a better predictor of incident diabetes than BMI among women but not among men [12]. In the Atherosclerosis Risk in Communities (ARIC) Study of adults aged 40-64 years, BMI and WC showed similar associations in men, with WHR being the weakest predictor [9], in close agreement with our findings. However, in female ARIC participants all anthropometric measures showed similar associations with diabetes risk, in contrast to our findings. Taken together, the results of these studies and our data suggest that the measure of obesity that best predicts diabetes may be dependent on age and sex, and that waist circumference is a better predictor of diabetes in women.

The measurement of WC is recommended in the US National Institutes of Health clinical guidelines for the assessment or management of obesity [3]. However, its utility in clinical practice has been questioned because of the training required to ensure that reliable WC data are collected [1]; other weaknesses, including lack of consensus regarding the best thresholds for defining obesity in different populations, have been discussed [24]. Of the few prospective studies which have assessed whether the combined use of general obesity with measures of body fat distribution can improve prediction of diabetes, some have suggested that combined use does not provide additional information beyond any single measure [9, 13], as was observed in this study. In older men, there is no evidence that measurement of WC provides additional clinical information beyond that provided by BMI, and vice versa in women. The finding that $\mathrm{WC}$ is a far better predictor of diabetes than $\mathrm{BMI}$ in older women lends support to the use of WC in older women (and perhaps in women in general) to identify those at high risk of developing diabetes. Further study would be required, however, before this could be done reliably in clinical practice.

The cut-off point for WC recommended for use in clinical practice remains controversial [1]. The ATP III criteria define 'abdominally obese' as WC $>88 \mathrm{~cm}$ for women and $>102 \mathrm{~cm}$ for men. However, in the present study, ROC curve analysis showed that WC thresholds of about 92 and $100 \mathrm{~cm}$ in women and men, respectively, provided optimal discrimination for diabetes, at least as defined in terms of the sum of sensitivity and specificity. The results suggest that the threshold could be increased in women. However, for women sensitivity would be substantially lowered if this method for defining optimal 
Table 4 Sensitivity, specificity and positive predictive value (PPV) and percentage of cases identified using sex-specific cut-off points for BMI and WC to predict diabetes over 7 years

\begin{tabular}{|c|c|c|c|c|c|c|c|c|c|c|c|c|}
\hline \multirow[t]{2}{*}{ Variable } & \multicolumn{6}{|c|}{ Men $(n=3,519)$} & \multicolumn{6}{|c|}{ Women $(n=3,404)$} \\
\hline & $n$ & Cases & $\%$ & Sensitivity & Specificity & PPV (\%) & $n$ & Cases & $\%$ & Sensitivity & Specificity & PPV (\%) \\
\hline \multicolumn{13}{|c|}{ BMI $\left(\mathrm{kg} / \mathrm{m}^{2}\right)$} \\
\hline$\geq 25$ & 2,382 & 147 & 67.7 & 89.1 & 33.4 & 6.2 & 2270 & 114 & 66.7 & 89.1 & 34.2 & 5.0 \\
\hline$\geq 26$ & 1,968 & 139 & 55.9 & 84.2 & 45.5 & 7.1 & 1916 & 107 & 56.3 & 83.6 & 44.8 & 5.6 \\
\hline$\geq 27$ & 1,506 & 123 & 42.8 & 74.5 & 58.8 & 8.2 & 1583 & 99 & 46.1 & 77.3 & 54.7 & 6.3 \\
\hline$\geq 28$ & 1,107 & 99 & 31.5 & 60.0 & 69.9 & 8.9 & 1290 & 92 & 37.9 & 71.9 & 63.4 & 7.1 \\
\hline$\geq 29$ & 799 & 89 & 22.7 & 53.9 & 78.8 & 11.1 & 1024 & 84 & 30.1 & 65.6 & 71.3 & 8.2 \\
\hline$\geq 30$ & 519 & 71 & 14.7 & 43.0 & 86.6 & 13.7 & 812 & 78 & 23.9 & 60.9 & 77.6 & 9.6 \\
\hline \multicolumn{13}{|c|}{ Waist (cm) } \\
\hline$\geq 80$ & & & & & & & 2,189 & 120 & 64.3 & 93.8 & 36.8 & 5.5 \\
\hline$\geq 81$ & & & & & & & 2,071 & 118 & 60.8 & 92.2 & 40.4 & 5.7 \\
\hline$\geq 82$ & & & & & & & 1,949 & 116 & 57.3 & 90.6 & 44.0 & 6.0 \\
\hline$\geq 83$ & & & & & & & 1,833 & 112 & 53.9 & 87.5 & 47.5 & 6.1 \\
\hline$\geq 84$ & & & & & & & 1,712 & 110 & 50.3 & 85.9 & 51.1 & 6.4 \\
\hline$\geq 85$ & & & & & & & 1,597 & 109 & 46.9 & 85.2 & 54.6 & 6.8 \\
\hline$\geq 86$ & & & & & & & 1,494 & 104 & 43.9 & 81.3 & 57.6 & 7.0 \\
\hline$\geq 87$ & & & & & & & 1,389 & 101 & 40.8 & 78.9 & 60.7 & 7.3 \\
\hline$\geq 88$ & & & & & & & 1,283 & 98 & 37.7 & 76.6 & 63.8 & 7.6 \\
\hline$\geq 89$ & & & & & & & 1,174 & 96 & 34.5 & 75.0 & 67.1 & 8.2 \\
\hline$\geq 90$ & & & & & & & 1,071 & 93 & 31.5 & 72.7 & 70.1 & 8.7 \\
\hline$\geq 91$ & & & & & & & 975 & 92 & 28.6 & 71.9 & 73.0 & 9.4 \\
\hline$\geq 92$ & & & & & & & 890 & 89 & 26.1 & 69.5 & 75.5 & 10.0 \\
\hline$\geq 93$ & & & & & & & 799 & 85 & 23.5 & 66.4 & 78.2 & 10.6 \\
\hline$\geq 94$ & 2,110 & 138 & 60.0 & 83.6 & 41.2 & 6.5 & 723 & 76 & 21.2 & 59.4 & 80.3 & 10.5 \\
\hline$\geq 95$ & 1,969 & 133 & 55.9 & 80.6 & 45.3 & 6.8 & 660 & 74 & 19.4 & 57.8 & 82.1 & 11.2 \\
\hline$\geq 96$ & 1,826 & 128 & 51.9 & 77.6 & 49.4 & 7.0 & & & & & & \\
\hline$\geq 97$ & 1,676 & 123 & 47.6 & 74.5 & 53.6 & 7.3 & & & & & & \\
\hline$\geq 98$ & 1,499 & 119 & 42.6 & 72.1 & 58.8 & 7.9 & & & & & & \\
\hline$\geq 99$ & 1,335 & 112 & 37.9 & 67.9 & 63.5 & 8.4 & & & & & & \\
\hline$\geq 100$ & 1,202 & 106 & 34.2 & 64.2 & 67.3 & 8.8 & & & & & & \\
\hline$\geq 101$ & 1,071 & 99 & 30.4 & 60.0 & 71.0 & 9.2 & & & & & & \\
\hline$\geq 102$ & 931 & 91 & 26.5 & 55.2 & 75.0 & 9.8 & & & & & & \\
\hline
\end{tabular}

threshold was applied and a higher threshold of $92 \mathrm{~cm}$ was used. The threshold of $88 \mathrm{~cm}$ yielded $76 \%$ sensitivity (specificity 63\%) whilst a threshold of $92 \mathrm{~cm}$ would yield a sensitivity of under $70 \%$ but a specificity of $75 \%$. By contrast in men, the results suggest that for diabetes prediction the current recommended WC guidelines of $102 \mathrm{~cm}$ could be lowered to $100 \mathrm{~cm}$ for identifying diabetes risk, supporting the findings of Wang et al. [10]. By lowering the threshold, sensitivity is increased though specificity decreases. This method of calculating optimal cut-points gives equal weighting to the notion of sensitivity and specificity and may not be ideal for the assessment of screening thresholds for diabetes in clinical practice. It is likely that optimal thresholds will depend on weighing the issues of cost as well as maximising the identification of those at high risk. We appreciate the need for replication of our findings in independent studies before changes to thresholds are made.

Our study is not without some limitation. It was carried out in an older, predominantly white, European population; we cannot generalise our findings to younger populations or to other ethnic groups, in which the relations between anthropometric measurements and diabetes risk are clearly different [25]. The determination of diabetes incidence in this study relied on documented doctor-diagnosed cases of diabetes, which inevitably results in under ascertainment of cases. This may also influence the performance of anthropometric measurements as BMI is the more routine marker measured in clinical practice. However, if anything, WC 
emerges as the far stronger predictor in women and this argument would bias against WC being a better predictor. Conversely it might be argued that since markers of central obesity (WC or WHR) have been shown to be more strongly associated with CHD risk than BMI in women in several studies [7, 26], women with higher WC are more likely to have health problems and more contact with their doctors and thus are more likely to be diagnosed. To address this possible bias we examined cross-sectionally the relationship of WC, BMI and hyperglycaemia (fasting glucose concentration $\geq 6.2 \mathrm{mmol} / \mathrm{l}$ ) in non-diabetics. WC was a significantly stronger predictor of hyperglycaemia than BMI in women, but in men WC and BMI showed similar predictive power. These findings suggest ascertainment bias is unlikely to explain $\mathrm{WC}$ being a better predictor of clinically diagnosed diabetes in women. The stronger association between WC and fasting blood glucose (a strong determinant of diabetes) compared with BMI in women may contribute to explaining why $\mathrm{WC}$ is a better predictor of diabetes than BMI. However, WC remained a significantly better predictor than BMI after adjustment for fasting blood glucose. Finally, our study cannot determine why WC should be a better predictor of diabetes in elderly women compared with elderly men, but suggests this as an area for future investigation.

\section{Conclusion}

Both overall and central adiposity indices are strong predictors of type 2 diabetes in older adults. BMI is as strong as WC in predicting type 2 diabetes in men. In women, however, WC was a significantly better measure for the identification of diabetes risk. The use of BMI combined with WC does not improve identification of type 2 diabetes risk beyond single measures of BMI (in men) or WC (in women). Current recommended guidelines for WC in men of $102 \mathrm{~cm}$ could be lowered to identify diabetes risk, whereas those for older women could be increased. Finally, future simple diabetes-prediction algorithms could usefully include WC in those applicable to women, though for changes to clinical practice one would need to ensure that better prediction in research settings is not negated by poorer reliability of the measurement in routine practice.

Acknowledgements The British Regional Heart Study is a British Heart Foundation (BHF) Research Group and is supported by a BHF Programme grant. The British Women's Heart and Health Study is jointly funded by the UK Department of Health and the BHF. O. Papacosta is supported by a project grant from Diabetes UK. The views expressed in this publication are those of the authors and not necessarily those of the funding bodies.

Duality of interest The authors declare that there is no duality of interest associated with this manuscript.
Open Access This article is distributed under the terms of the Creative Commons Attribution Noncommercial License which permits any noncommercial use, distribution, and reproduction in any medium, provided the original author(s) and source are credited.

\section{References}

1. Klein S, Allison DB, Heymsfield SB, Association for Weight Management and Obesity Prevention; NAASO; Obesity Society; American Society for Nutrition; American Diabetes Association; Association for Weight Management and Obesity Prevention; NAASO, the Obesity Society; the American Society for Nutrition; and the American Diabetes Association et al (2007) Waist circumference and cardiometabolic risk: a consensus statement from shaping America's health. Diabetes Care 30:1647-1652

2. Gallergher D, Visser M, Sepulveda D, Pierson RN, Harris T, Heymsfield SB (1996) How useful is body mass index for comparison of body fatness across age, sex and ethnic groups? Am J Epidemiol 143:228-239

3. National Institutes of Health (1998) Clinical guidelines on the identification, evaluation and treatment of overweight and obesity in adults - the evidence report. Obes Res 6:51S-209S

4. Wei M, Gaskill SP, Haffner SM, Stern MP (1997) Waist circumference as the best predictor of noninsulin dependent diabetes mellitus (NIDDM) compared to body mass index, waist/hip ratio and other anthropometric measurements in Mexican Americans - a 7-year prospective study. Obes Res 5:16-23

5. The Diabetes Prevention Program Research Group (2006) The relationship of body size and shape to the development of diabetes in the Diabetes Prevention Program. Obesity 14:2107-2117

6. Snijder MB, Dekker JM, Visser M et al (2003) Associations of hip and thigh circumferences independent of waist circumference with the incidence of type 2 diabetes: the Hoorn Study. Am J Clin Nutr 77:1192-1197

7. Folsom AR, Kushi LH, Anderson KE et al (2000) Associations of general and abdominal obesity with multiple health outcomes in older women: the Iowa Women's Health Study. Arch Intern Med 160:2117-2128

8. Carey VJ, Walters EE, Colditz GA et al (1997) Body fat distribution and risk of non-insulin-dependent diabetes mellitus in women. The Nurses' Health Study. Am J Epidemiol 145:614619

9. Stevens J, Couper D, Pankow J et al (2001) Sensitivity and specificity of anthropometrics for the prediction of diabetes in a biracial cohort. Obes Res 9:696-705

10. Wang Y, Rimm EB, Stampfer MJ, Wilett WC, Hu FB (2005) Comparison of abdominal adiposity and overall obesity in predicting risk of type 2 diabetes among men. Am J Clin Nutr $81: 555-563$

11. Meisinger C, Döring A, Thorand B, Heier M, Löwel H (2006) Body fat distribution and risk of type 2 diabetes in the general population: are there differences between men and women? The MONICA/KORA Augsburg Cohort Study. Am J Clin Nutr $84: 483-489$

12. Schulze MB, Heidemann C, Schienkiewitz A, Bergmann MM, Hoffmann K, Boeing H (2006) Comparison of anthropometric characteristics in predicting the incidence of type 2 diabetes in the EPIC-Potsdam Study. Diabetes Care 29:1921-1923

13. Tulloch-Reid MK, Williams DE, Looker HC, Hanson RL, Knowler WC (2003) Do measures of body fat distribution provide information on the risk of type 2 diabetes in addition to measures of general obesity? Comparison of anthropometric predictors of type 2 diabetes in Pima Indians. Diabetes Care 26:2556-2561 
14. Vazquez G, Duval S, Jacobs DR Jr, Silventoinen K (2007) Comparison of body mass index, waist circumference, and waist/ hip ratio in predicting incident diabetes: a meta-analysis. Epidemiol Rev 29:115-128

15. Wild S, Roglic G, Green A, Sicree R, King H (2004) Global prevalence of diabetes: estimates for the year 2000 and projections for 2030. Diabetes Care 27:1047-1053

16. Shaper AG, Pocock SJ, Walker M, Cohen NM, Wale CJ (1981) British Regional Heart Study: cardiovascular risk factors in middle-aged men in 24 towns. BMJ 283:179-186

17. Walker M, Shaper AG, Lennon L, Whincup PH (2000) Twenty year follow-up of a cohort study based in general practices in 24 British towns. J Publ Health Med 22:479-485

18. Lawlor DA, Bedford C, Taylor M, Ebrahim S (2003) Geographical variation in cardiovascular disease, risk factors, and their control in older women: British Women's Heart and Health Study. J Epidemiol Community Health 57:134-140

19. Wannamethee SG, Lowe GDO, Whincup PH, Rumley A, Walker M, Lennon L (2002) Physical activity and hemostatic and inflammatory variables in elderly men. Circulation 105:17851790
20. Emberson J, Whincup PH, Walker M, Thomas M, Alberti KGMM (2002) Biochemical measures in a population based study: the effect of fasting duration and time of day. Ann Clin Biochem 39:493-501

21. Lawlor DA, Sattar N, Davey Smith G, Ebrahim S (2005) The associations of physical activity and adiposity with alanine aminotransferase and gamma-glutamyltransferase. Am J Epidemiol 161:1081-1088

22. Perry IJ (1995) Non-insulin-dependent diabetes and coronary heart disease in middle-aged British men. $\mathrm{PhD}$ thesis, University of London

23. DeLong ER, DeLong DM, Clarke-Pearson DL (1988) Comparing the areas under two or more correlated receiver operating characteristic curves: a nonparametric approach. Biometrics 44:837-845

24. Han TS, Sattar N, Lean M (2006) ABC of obesity. Assessment of obesity and its clinical implications. BMJ 333:695-698

25. WHO Expert Consultation (2004) Appropriate body-mass index for Asian populations and its implications for policy and intervention strategies. Lancet 363:157-163

26. Yang L, Kuper H, Weiderpass E (2008) Anthropometric characteristics as predictors of coronary heart disease in women. $\mathrm{J}$ Intern Med 264:39-49 